\title{
Corrigendum: Long non-coding RNAs and enhancer RNAs regulate the lipopolysaccharide-induced inflammatory response in human monocytes
}

Nicholas E. Ilott, James A. Heward, Benoit Roux, Eleni Tsitsiou, Peter S. Fenwick, Luca Lenzi, lan Goodhead, Christiane Hertz-Fowler, Andreas Heger, Neil Hall, Louise E. Donnelly, David Sims \& Mark A. Lindsay

Nature Communications 5:3979 doi: 10.1038/ncomms4979 (2014); Published 9 Jun 2014; Updated 9 Apr 2015

The original version of this Article contained a typographical error in the spelling of the author Nicholas E. Ilott, which was incorrectly given as Nicholas E. IIott. This has now been corrected in both the PDF and HTML versions of the Article. 